\title{
Evolution of Materials Properties and Macropores in Thermally Crept Nuclear Graphite
}

\author{
Joshua J. Kane ${ }^{1 *}$, Austin C. Matthews ${ }^{2}$, Timothy Yoder $^{2}$ and William E. Windes ${ }^{2}$ \\ 1. Advanced Post-Irradiation Examination Department, Materials and Fuels Complex, Idaho National \\ Laboratory, Scoville, Idaho, USA. \\ 2. Materials Science \& Engineering Department, Energy and Environmental Science and Technology, \\ Idaho National Laboratory, Idaho Falls, Idaho, USA. \\ * Corresponding author: joshua.kane@inl.gov
}

Graphite is an important component to the design of high-temperature gas-cooled reactors. Many of these reactors use graphite as a neutron moderator and/or structural component such as fuel- and gascoolant channels [1], [2], [3]. Graphite is a desirable material because of its low neutron absorption cross-section and its high-temperature strength [4]. During operation, material properties and dimensions can change as a function of neutron irradiation dose and temperature. Gradients in both temperature and dose can lead to significant stress and distortion of the graphite components. Over time these stresses and distortions, if unaccounted for, can lead to failure of components, blockage of coolant channels, and even restriction of control rod sleeves [5].

Irradiation-induced changes in graphite are known to be strongly linked to as fabricated properties of nuclear graphite. While multiple efforts are underway worldwide to correlate irradiation dose, temperature, and property changes, progress is limited due to: (1) the expense of irradiation experiments, (2) limited number of research reactors for accelerated testing and the inherent limited space (few samples per experiment) leading to greater uncertainty and (3) the length of time needed in a test reactor to reach the desired irradiation damage (years). Additionally, may important irradiation phenomena, such as irradiation creep, have both a microstructural and crystallographic component and deconvolution of their contributions at high levels of neutron irradiation damage has proved challenging via irradiation experiments alone.

To better understand the microstructural influence of irradiation damage on graphite irradiation creep and thermo-mechanical changes, Idaho National Laboratory has investigated the thermal creep of graphite. Incremental thermal creep experiments were conducted over a temperature range of $1500^{\circ} \mathrm{C}$ to $2600^{\circ} \mathrm{C}$ in a high temperature graphite furnace modified to accommodate sample loads (compression) between 0 and $60 \mathrm{MPa}$. The thermal creep behavior of two graphite grades, NBG-18 (SGL, Germany) and PCEA (Graftech, USA) candidate nuclear graphites, respectively, were examined over a range of conditions encompassing primary and secondary creep regimes. After each experimental increment samples were characterized in terms of their dimensions, mass, thermal diffusivity, coefficient of thermal expansion, and Young's modulus. Multiple samples were also non-destructively examined via $\mu \mathrm{X}$-ray Computed Tomography to quantitatively track changes in microstructure and feature morphology throughout the life of the experiment.

This work focuses on the quantification of the interrupted thermal creep $\mu X$-ray Computed Tomography experiments. More specifically in the region traditionally labeled primary creep. Although this region varies somewhat with temperature and load, it occurs within the first several hours of the experiment. During this time graphite can shrink in size by $3-5 \%$ in the axial direction. Furthermore, the total sample 
volume is not conserved during primary creep of nuclear graphite. After observing this large dimensional change under volumetrically non-conservative conditions, it was speculated that traditional primary creep in graphite under the experimental conditions examined is the plastic collapse of macroporosity within nuclear graphite. To confirm this, successive 3D volumes were analyzed via digital volume correlation. A representative case is shown in Figure 1. The overlaid displacement field shows the vast majority of displacement occurring in the immediate vicinity of large macropores. Changes to macroporosity was further examined in terms of its effective path length, interconnectivity, and size. A representation of changing effective path length is shown in Figure 2.

This work has yielded additional insight into the origin of "primary" creep in graphite. Rather than true thermal creep, the large observed deformation appears to come from the plastic collapse of pores.

\section{References:}

[1] BJ Marsden and LH Fok, International Conference on Structural Mechanics in Reactor Technology (SMiRT) (2005) p. 7.

[2] R Bratton and W Windes, INL/MIS-10-19427, Idaho National Laboratory (2010).

[3] W Windes, T Burchell and R Bratton, INLEXT-07-13165, Idaho National Laboratory (2011).

[4] BT Kelly in "Physics of Graphite", (Academic Press, New York and London).

[5] BT Kelly, BJ Marsden and K Hall, TECDOC-1154, International Atomic Energy Agency (2000).
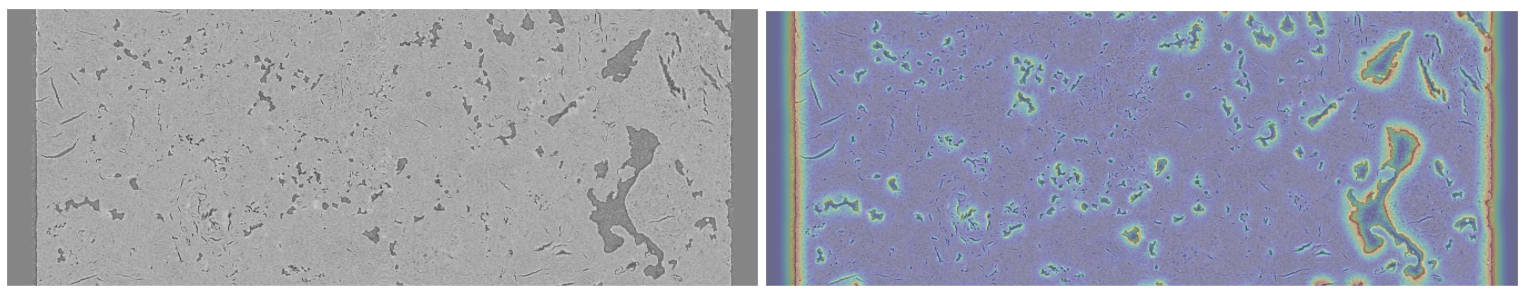

Figure 1. The left image is an axial cross-section of an NBG-18 sample and the rightmost image is the same image with a displacement field overlay. Red corresponds to the highest displacement $(\sim 31 \mu \mathrm{m})$.

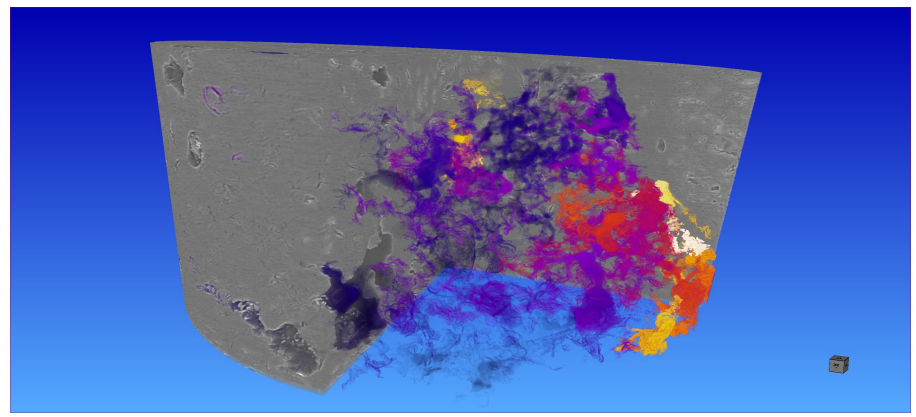

Figure 2. The leftmost image shows a 3D representation of an NBG-18 graphite reconstructed sample in gray. The color region represents the effective path length change within a sub-section of the volume analyzed. 